\title{
SUR QUELQUES PHLEBOTOMES DE YOUGOSLAVIE
}

\author{
Par Virgil NITZULESCU
}

Comme nous poursuivons des études systématiques et anatomiques'sur les phlébotomes européens, nous nous sommes adressés à plusieurs savants étrangers, en les priant de nous envoyer du matériel. Or, dans un lot de phlébotomes que le $\mathrm{D}^{r}$ Tchédomir Simitch, de Skoplje, a bien voulu nous adresser (1), nous venons de trouver des formes de phlébotomes qui présentent un intérêt particulier et qui feront l'objet de cette note.

Le lot comprenait un exemplaire mâle typique de Phlebotomus perniciosus Newst. que nous ne décrirons pas ici, trois exemplaires mâles, ressemblant au premier abord à $P$. perniciosus, mais différant de celui-ci par des caractères qui seront exposés dans la suite, le reste du lot étant formé par des femelles dont nous donnerons de même la description plus bas.

Nous commencerons par la description des trois mâles. Nous donnerons des chiffres en microns représentant les différentes dimensions des organes quoique l'expérience acquise en mesurant une grande quantité de phlébotomes finit vite par convaincre combien ces chiffres sont variables, tant d'une manière absolue que relative. Souvent, par exemple, les deux antennes du même individu, les nervures correspondantes des deux ailes, etc., varient entre elles comme dimensions. Toutefois, en général, ces variations ne dépassent pas certaines limites et, comme nos trois exemplaires ont sensiblement une taille égale, en présentant les chiffres extrêmes, le plus petit et le plus grand pour un même organe, nous espérons donner une idée suffisante des dimensions respectives. Malheureusement, nous ne pouvons pas faire pour tous les organes la même chose, parce que plusieurs de nos phlébotomes ont perdu en cours de voyage une partie de leurs antennes et de leurs pattes. Dans ce cas, nous avons choisi l'exemplaire qui gardait l'organe en entier et nous n'avons mesuré cet organe que chez celui-ci.

(1) Nous exprimons ici encore une fois toute notre reconnaissance à notre ancien camarade du Laboratoire de parasitologie de la Faculté de médecine de Paris, le $\mathrm{D}^{\mathrm{r}}$ Simitch, à qui nous avons tant d'obligation pour son précieux envoi.

Annales de Parasitologie, T. VII, $\mathrm{N}^{\circ} 6 .-1^{\mathrm{er}}$ novembre 1929 , p. 494-505. 


\section{DEscription DES MALES}

\section{Dimensions}

Tête et clypeus

Thorax

Abdomen

$1^{\text {er }}$ Segment de la gonapophyse supérieure

Total
395-405 $\mu$

ธ50-575 $\mu$

820-980 $\mu$

270-300 $\mu$

$2.035-2.260 \mu$

\section{Antennes}

Un seul exemplaire gardait les antennes entières. Voici les dimensions des différents segments de son antenne gauche :

Segm. I ........... $72 \mu$

Segm. II ............ $72 \mu$

Segm. III $\ldots \ldots \ldots \ldots \ldots \ldots .280 \mu$

Segm. IV ............ $123 \mu$

Segm. V . . . . . . . . $123 \mu$

Segm. VI ........... $122 \mu$

Segm. VII $\ldots \ldots \ldots \ldots \ldots \ldots .118 \mu$

Segm. VIII .......... $112 \mu$

Segm. IX $\ldots \ldots \ldots \ldots \ldots \ldots \quad 110 \mu$

Segm. X ............ $105 \mu$

Segm. XI ........... $103 \mu$

III $>$ IV + V

III $<$ IV + V + VI

III $<$ XII-XVI

$\mathrm{IV}+\mathrm{V}<\mathrm{XII}-\mathrm{XVI}$

$\mathrm{IV}+\mathrm{V}+\mathrm{VI} \geq \mathrm{XII-XVI}$

Segm. XII ........... $92 \mu$

Segm. XIII .......... $75 \mu$

Segm. XIV ........... $65 \mu$

Segm. XV ............. $61 \mu$

Segm. XVI ........... $68 \mu$

Total de l'antenne : $1.701 \mu$, donc un millimetre trois quarts environ.

\section{Palpes}

Segm. I ............ $40 \mu$

Segm. II . . . . . . . . 140-150 $\mu$

Segm. III .......... $155-160 \mu$

Segm. IV . . . . . . 112-130 $\mu$

Segm. V $\ldots \ldots \ldots \ldots .290-355 \mu$
Formule palpale : $1,4,2,3,5$.

V $>$ III + IV

$\mathrm{V}<\mathrm{III}+\mathrm{IV}+$ II

$\mathrm{V}= \pm \mathrm{IV} \times 2,5$

\section{Pattes}

Un seul exemplaire a été mesuré ; taille de cet exemplaire: $2.050 \mu$. 
Première paire de pattes :

Fémur ............. $\quad 690$ H

Tibia ............... $\quad \mathbf{7 4 0} \mu$

Tarse $1 \ldots \ldots \ldots \ldots .6 .480 \mu$

Tarse $2 \ldots \ldots \ldots \ldots . .250 \mu$

Tarses $3-5 \quad \ldots \ldots \ldots \ldots . \quad 370$

$\frac{\text { Tarse } 1}{\text { Tarse } 2}=1,9 \quad \frac{2350}{2050}=1,1$

Total

$2.530 \mu$

Deuxième paire de pattes :

Fémur ............. $\quad 690 \mu$

Tibia .............. $\quad 890 \mu$

Tarse $1 \ldots \ldots \ldots \ldots . .650 .6$

Tarse $2 \ldots \ldots \ldots \ldots . .285 \mu$

Tarses $3-5 \ldots \ldots \ldots \ldots . \quad 380$

$\frac{\text { Tarse } 1}{\text { Tarse } 2}=1,9 \quad, \frac{2810}{2050}=1,3$

, Total .......... $2.810 \mu$

Troisième paire de pattes :

Fémur

Tibia

Tarse

Tarse 2

Tarses 3-5

Total
$780 \mu$

$1.075 \mu$

$655 \mu$

$325 \mu$

$450 \mu$ $\frac{\text { Tarse } 1}{\text { Tarse } 2}=2,01$

Aile

Le bord postérieur de l'aile est plus arqué que le bord antérieur. Longueur de l'aile . 1.750-1.850 $\mu$ Largeur maxima ..

$$
\begin{array}{r}
500-510 \mu \\
350-380 \mu \\
206-210 \mu \\
85-100 \mu
\end{array}
$$

$$
\frac{\alpha}{\beta} 1,70-1,80
$$

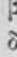

\section{Armature génitale}

En général, au type major ou perniciosus, cinq longues épines sur le deuxième segment de la gonapophyse supérieure. Une touffe de poils sur le premier segment au milieu de sa face interne (fig. 1).

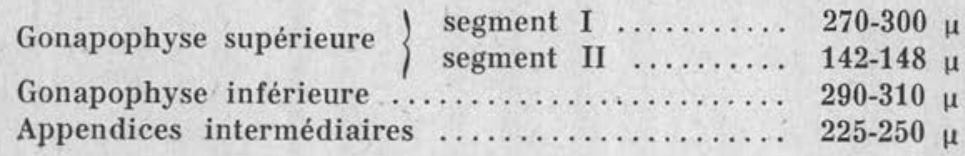


L'organe intromittent présente une extrémité arrondie et un tubercule subapical inférieur. La pompe génitale mesure 90-110 $\mu$. Elle est située chez un exemplaire dans le troisième segment, chez les deux autres dans le quatrième. Les filaments génitaux sortent loin de l'extrémité distale de l'organe intromittent. Leur longueur est de 680-760 $\mu$.

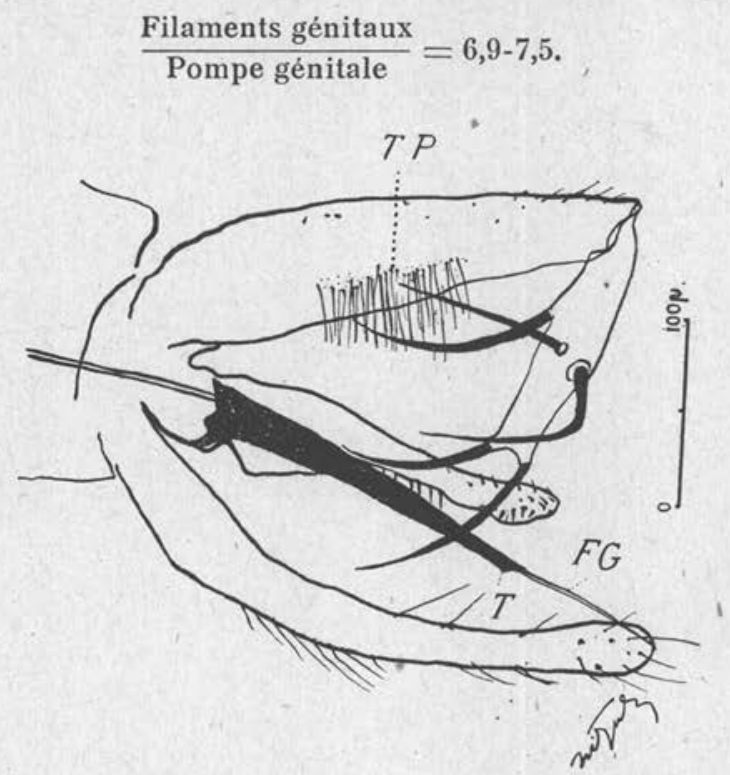

Fig. 1. - Armature gênitale d'un des trois phlébotomes mâles de Skoplje (lot du Dr Simitch). TP, touffe de poils; T, tubercule subapical inférieur de l'organe intromittent ; FG, filaments génitaux.

Discussion. - L'intérêt de ces trois exemplaires consiste dans le fait que tous les trois présentent des filaments génitaux très longs (fig. 2). Si on compare la longueur de la pompe à la longueur des filaments génitaux, on voit que ceux-ci sont au moins 7 fois plus longs. Chez $P$. perniciosus, les filaments génitaux sont très courts et la pompe est située dans les tout derniers segments de l'abdomen.

Le deuxième caractère important, chez ces trois phlébotomes, est que l'organe intromittent n'est pas taillé en biseau, ni bifide à l'extrémité, et présente en échange un tubercule subapical inférieur.

Ces deux caractères s'opposent à l'identification des phlébotomes de Skoplje avec le $\boldsymbol{P}$. perniciosus Newst. et les rapprochent dans la même mesure de $P$. major var. longiductus de Parrot, phlébotome dont l'existence a déjà été signalée par nous en Europe et notamment en Roumanie dans un article précédent.

Annales de Parasitologie, T. VII, No $6 .-1^{\mathrm{er}}$ novembre 1929.

32. 
Le type de Skoplje et celui que nous avons décrit à Bucarest ne sont pas toutefois identiques. Certains détails les séparent. En voici quelques-uns :

Chez les phlébotomes de Skoplje, la touffe de poils de la gonapophyse supérieure est un peu moins marquée que chez l'exemplaire de Bucarest. L'organe intromittent est plus mince et plus long comparé à l'appendice intermédiaire. Son tubercule subapical est plus petit et un peu, plus antérieur. Les filaments génitaux, qui comme nous venons de le voir, sont bien plus longs que chez perniciosus, sont néanmoins plus courts que chez la variété longiductus de Bucarest, chez lequel ils mesurent presque dix fois la lon-
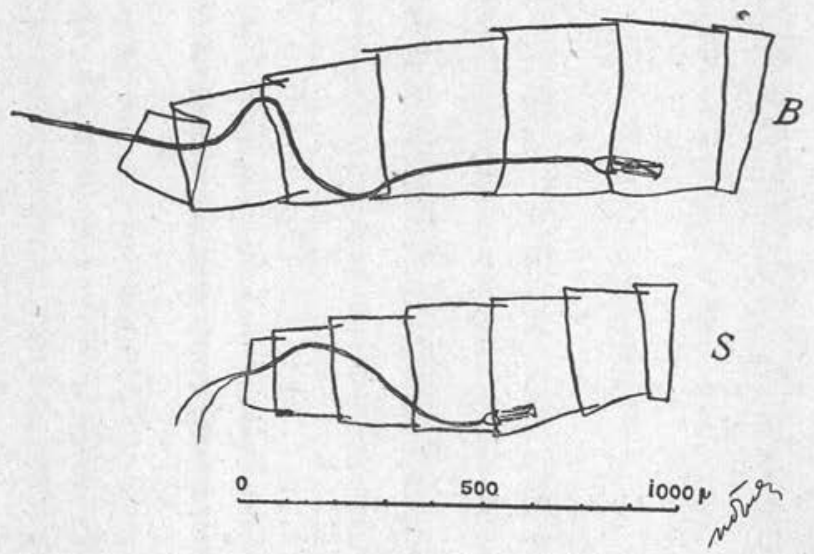

Fig. 2. - Abdomen du phlébotome mâle du type chinensis de Bucarest (B) comparé à celui de Yougoslavie (S). Les filaments génitaux sont dans le premier (B) presque dix fois aussi longs que la pompe, dans le second (S) seulement sept fois.

gueur de la pompe. Celle-ci était bien proximale dans le type de Bucarest (fig. 2). Elle arrive à peine jusqu'au troisième segment chez l'un des trois exemplaires de Yougoslavie, dans le $4^{\circ}$ chez les deux autres.

De tous ces caractères, c'est naturellement la moindre longueur des filaments génitaux qui sépare le plus les deux types. La position de la pompe en elle-même a une importance relative car' cet organe n'a pas une position fixe (du moins jusqu'à un certain point) chez un même individu. Si les filaments génitaux sortent par le pénis, la pompe descend dans la même mesure, et chez tous les exemplaires de Skoplje les filaments sortent. En ce qui concerne la longueur des filaments, certains phlébotomes d'Asie que Perfiliev identifie toutefois avec le $P$. major var. longiductus, présen- 
tent des filaments encore plus courts, 6 fois la longueur de la pompe (fig. 24 de Perfiliev, loc. cit.); cette iongueur est bien supérieure à celle de $P$. major ou de sa variété perniciosus.

Bref, nous nous trouvons en présence d'un type un peu différent de phlébotome à conduitśs génitaux très longs, que nous devons étiqueter, provisoirement au moins, comme $P$. major var. longiductus, à cause de la forme de l'organe infromittent et de la longueur relative des filaments génitaux. Mais la discussion ne finit pas ici.

Nous avons mentionné, dans notre précédent article, que Sinton reconnaît dans l'exemplaire décrit par Parrot sous le nom nouveau de longiductus, un mâle de $P$. chinensis, parce qu'il « présente le même organe intromittent et les mêmes filaments génitaux ». Or, les filaments dủ mâle de Parrot étaient excessivement longs et la pompe était bien proximale. Les phlébotomes de Skoplje doivent-ils eux aussi entrer dans la catégorie indiquée par Sinton, quoique leurs filaments génitaux soient moins longs ? Ou doit-on les mettre dans une catégorie à part, avec les longiductus décrits par Perfiliev? En d'autres termes: les filaments génitaux de $P$. chinensis, doivent-ils nécessairement être excessivement longs ? Comme nous ne trouvons aucune description détaillée du mâle de $P$. chinensis, tâchons de reconstituer l'histoire de cette espèce.

Le Phlebotomus chinensis a été séparé de l'espèce asiatique $P$. major par Newstead, en 1916, sous la forme d'une simple variété de celui-ci : $P$. major var. chinensis. Les caractères qui la différenciaient du type $\boldsymbol{P}$. major, étaient, dans la description de Newstead, très peu importants : longueur plus considérable du segment proximal de la gonapophyse supérieure, aplatissement à l'extrémité des cinq épines de cette gonapophyse et égalité des articles 2,3 et 4 des palpes du mâle. Plus récemment, Patton et Hindle (1926) lui attribuent des caractères nouveaux : formule palpale $1,4,2,3,5$; premier article du tarse trois fois plus long que le second et divers caractères de la nervation de l'aile.

C'est plus récemment encore, à la suite des très intéressantes recherches de Sinton et Barraud, d'Adler et Theodor, qu'on vient de séparer la variété chinensis comme une espèce à part, bien distincte de $P$. major. Ces auteurs ont observé que chez les phlébotomes en général, la forme des spermathèques et de l'armature pharyngienne de la femelle sont d'un grand secours dans la systématique. A l'inverse de ce qu'on croyait avant eux, les phlébotomes peuvent souveht se caractériser bien mieux par la femelle que par le mâle ; les caractères des mâles avaient fait du Phlebotomus chinensis une variété de $P$. major; les caractères des femelles imposent la création d'une espèce indépendante. Les voici : 
La spermathèque de $P$. major est largement crénelée sur les bords et se continue par un long cou suivi d'une petite tête; celle de $P$. chinensis est fusiforme, à parois lisses, à col extrêmement court et présente, à sa surface, des lignes qui s'entrecroisent en circonscrivant des aires triangulaires.

Les conduits des spermathèques se terminent séparément chez $P$. chinensis, ils s'unissent à leur terminaison chez $P$. major.

L'armature pharyngienne de la femelle de $P$. major est formée de très petites dents groupées en petites séries linéaires, séparées les unes des autres et donnant l'impression de lignes pointillées souvent interrompues; celle de la femelle de $P$. chinensis est formée de deux zones: une zone antérieure de dents assez larges, encerclée en arrière par une autre zone de lignes transversales.

Tous ces caractères sont bien nets et faciles à observer. Ce sont eux qui nous aideront à trancher la question des phlébotomes douteux d'Europe.

En effet, si les femelles des phlébotomes à conduits génitaux excessivement longs (rapport $\frac{\text { filaments }}{\text { pompe }}=10,1$ ) présentent les mêmes spermathèques et la même armature pharyngienne que les femelles des phlébotomes mâles à conduits génitaux un peu plus courts (rapport $\frac{\text { filaments }}{\text { pompe }}=7,1$ ou 6,1 ), il serait hors de doute que la position de la pompe et la longueur des conduits sont des caractères variables dans le sein d'une même espèce; si les caractères des femelles mentionnés plus haut sont justement ceux qui ont été découverts par Sinton et Barraud, Adler et Theodor dans l'espèce chinensis, nous pourrons affirmer la présence, en Europe, de cette espèce bien plus sûrement encore que nous n'avons pu le faire par la description des mâles seuls, d'autant plus qu'une partie de ces mâles ne se superposaient pas exactement aux mâles considérés par Sinton comme des $P$. chinensis.

Avant de continuer cette discussion, examinons donc de plus près les femelles du lot envoyé par le $\mathrm{D}^{\mathrm{r}}$ Simitch et donnons leur description.

\section{DESCRIPTION DES FEMELLES}

Les femelles ont été gonflées par la potasse, afin de mieux voir les spermathèques. Les chiffres qui suivront sont donc un peu plus élevés qu'ils n'auraient été si nous avions monté simplement les insectes dans le baume. 


\section{Dimensions}

Tête et clypeus $\ldots \ldots \ldots \ldots \ldots \ldots \ldots \ldots \ldots \ldots . .6 \ldots \ldots$

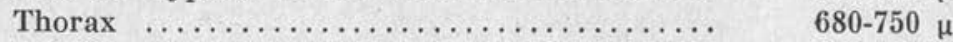

Abdomen (les appendices supérieurs inclus) $\ldots \quad 1.525-1.750 \mu$

Total $\ldots \ldots \ldots \ldots \ldots \ldots \ldots \ldots \ldots \ldots \ldots 2.630-2.950 \mu$

\section{Antennes}

Segm. I $\ldots \ldots \ldots$.

Segm. II $\ldots \ldots \ldots$.

Segm. III $\ldots \ldots$. .

Segm. IV .........

Segm. V ........

Segm. VI .......

Segm. VII ........

Segm. VIII .......

Segm. IX $\ldots \ldots \ldots$.

Segm. X ........

Segm. XI .........

Segm. XII .........

Segm. XIII ........

Segm. XIV .........

Segm. XV .........

Segm. XVI ........

$$
\text { Total } \ldots \ldots \ldots \overline{1.640-1.736 \mu}
$$

\section{Palpes}

Segm. I $\ldots \ldots \ldots \ldots \ldots . \quad 40-50 \mu$ Formule palpale : 1, 4, 2, 3, 5

Segm. II $\ldots \ldots \ldots \ldots \ldots .170-190 \mu$

Segm. III $\ldots \ldots \ldots \ldots \ldots$ 180-190 $\mu$

Segm. IV ......... $130-150 \mu$

III $>$ IV + V

106-120 $\mu$

101-103 $\mu$

100-103 $\mu$

97-98 $\mu$

$90-98 \mu$

$89-90 \mu$

70-74 $\mu$

66-68 $\mu$

III $<$ IV + V + VI

III $<$ XII-XVI

IV + V $<$ XII-XVI

$\mathrm{IV}+\mathrm{V}+\mathrm{VI}<\mathrm{XII}-\mathrm{XVI}$

Segm. V

$370-430 \mu$

$\mathrm{V}>\mathrm{III}+\mathrm{IV}$

$1,4(2,3), 5$

$\mathrm{V}<\mathrm{II}+\mathrm{III}+\mathrm{IV}$

$\mathrm{V}>\mathrm{IV} \times 2,5$

\section{Pattes}

Première paire de pattes :

Fémur $\ldots \ldots \ldots \ldots \ldots . \quad 775-830 \mu$

Tibia ........... $880-890 \mu$

Tarse $1 \ldots \ldots \ldots \ldots .6 .515-540 \mu$

Tarse $2 \ldots \ldots \ldots \ldots .280-300 \mu$

$\frac{\text { Tarse } 1}{\text { Tarse } 2}=1,8$

Tarses $3-5 \ldots \ldots \ldots .400-430 \mu$ 
Deuxième paire de pattes :

Fémur ........... 735- $820 \mu$

Tibia $\ldots \ldots \ldots \ldots$. 1.005-1.065 $\mu$

Tarse 1 ....... $\quad 605-650 \mu$

Tarse $2 \ldots \ldots \ldots . \quad 305-325 \mu$

Tarses $3-5 \ldots \ldots . \quad 430-440 \mu$

$\frac{\text { Tarse } 1}{\text { Tarse } 2}=1,9-2,0$

Troisième paire de pattes :

Fémur .......... 900-940 $\mu$

Tibia ........... 1.320-1.370 $\mu$

Tarse $1 \ldots \ldots \ldots . \quad 780-805 \mu$

Tarse $2 \ldots \ldots \ldots . \quad 375-400 \mu$

Tarses $3-5 \ldots \ldots . \quad 507-527 \mu$

$\frac{\text { Tarse } 1}{\text { Tarse } 2}=2,01-2,08$

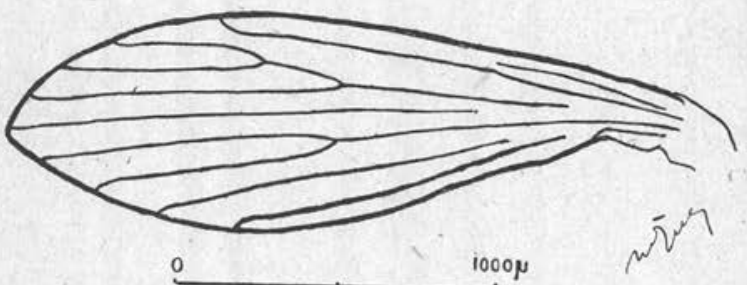

Fig. 3. - Aile d'une des femelles de Skoplje (lot du Dr Simitch).

Aile

Le bord postérieur est plus arqué que le bord antérieur (fig. 3) :

Longueur ....... 2.240-2.280 $\mu$

Largeur maxima...

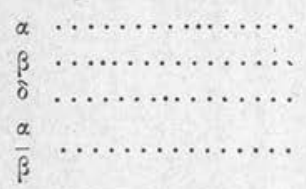

640-660 $\mu$

$420-490 \mu$

$250-275 \mu$ $100-150 \mu$

$1,53-1,81$
Quelques exemples de rapports :

$$
\begin{array}{ll}
\frac{\alpha}{\beta}=\frac{475}{265}=1,78 ; & \frac{\alpha}{\beta}=\frac{460}{255}=1,81 \\
\frac{\alpha}{\beta}=\frac{420}{275}=1,53 ; & \bar{\beta}=\frac{440}{275}=1,6
\end{array}
$$

\section{Spermathèques}

Les spermathèques (fig. 4) 'sont fusiformes, assez allongées et présentent

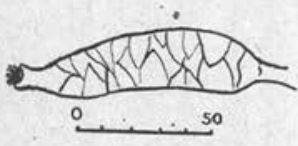

FIG. 4. - Spermathèque d'une des femelles de Skoplje (lot du Dr Simitch). à leur surface des lignes qui s'entrecroisent en circonscrivant des aires triangulaires. Le col extrêmement court. Les conduits sont assez longs et se terminent séparément. Ce sont là les caractères des spermathèques de $P$. chinensis, tels qu'ils ont été décrits tout récemment par les auteurs cités plus haut. 
Dimensions de la spermathèque :

Longueur ...................... $80-85 \mu$

Largeur maxima $\ldots \ldots \ldots \ldots \ldots \ldots \ldots \ldots . \quad 18-21 \mu$

\section{Armature pharyngienne}

Le pharynx (fig. 5), assez large, laisse voir à sa partie postérieure deux zones de dents distinctes : la première et la plus antérieure est triangulaire, avec la pointe en avant ; elle est formée de dents assez bien développées et noirâtres, ce qui fait qu'elle se distingue bien, même aux faibles grossissements. La deuxième zone, beaucoup plus pâle, est formée de lignes transversales, qui encadrent la première en arrière. Cette armature pharyngienne avec ses deux zones de dents correspond aussi aux descriptions du pharynx de $P$. chinensis. Il n'y a qu'à comparer la figure que nous en donnons (figure 5), avec les figures des auteurs anglais, pour s'en convaincre.

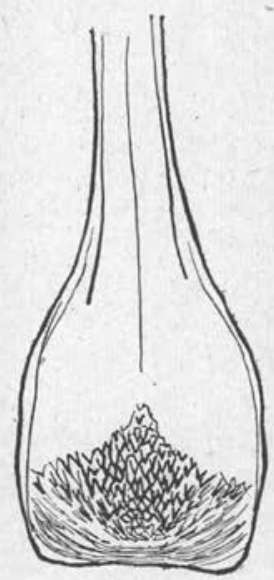

Fig. 5. - Armature pharyngienne d'une des femelles de Skoplje (lot du Dr Simitch).

Discussion. - Nous voilà donc en présence de femelles de phlébotomes capturées en Europe, et présentant des spermathèques et une armature pharyngienne caractéristique de l'espèce asiatique $P$. chinensis. Nous ne pouvons plus hésiter dans l'identification. Les femelles du lot du $\mathrm{D}^{\mathrm{r}}$ Simitch, au moins dans l'état actuel de la classification des phlébotomes, ne peuvent être considérées autrement que comme appartenant au P. chinensis. Quant aux mâles décrits plus haụt, tout nous porte maintenant à les considérer comme correspondant à ces femelles. En effet, comme nous l'avons vu, c'est de l'espèce chinensis qu'ils se rapprochent le plus. Le fait d'avoir des filaments génitaux un peu plus courts, fait qui n'avait pas empêché Perfiliev de considérer comme «longiductus 》 des phlébotomes semblables d'Asie, ne nous empêchera pas, nous aussi, de les considérer comme des $P$. chinensis, puisque nous avons trouvé à côté d'eux des femelles bien caractérisées comme telles.

Quant au phlébotome de Bucarest, nous hésitions dans notre publication antérieure à l'identifier avec le $P$. chinensis, bien que le mâle se superposât exactement à la description de Sinton, car nous n'avions pas encore trouvé de femelle correspondante, mais un fait nouveau vient de se produire. Une campagne de recherches, 
systématiquement poursuivie à Bucarest par Mme le $\mathrm{D}^{\mathbf{r}}$ G. Nitzulescu, a abouti enfin à la découverte d'un gîte dans lequel à peu près une centaine d'exemplaires mâles et femelles viennent a'être capturés. Tous les mâles de ce gîte, examinés jusqu'ici, étaient semblables à celui que nous avons décrit. Toutes les femelles présentaient l'armature pharyngienne et les spermathèques du $P$. chinensis (1).

De cet ensemble de faits d'observation s'appuyant mutuellement nous tirons les conclusions suivantes :

\section{RÉSUMÉ}

Le Phlebotomus major var. longiductus de Parrot est bien le $P$. chinensis Newst.

Nous venons de rencontrer cette espèce asiatique en Europe et notamment en Roumanie et en Yougoslavie. Nous avons caractérisé les femelles par la forme des spermathèques et de l'armature pharyngienne et les mâles par la forme de l'organe intromittent et par la longueur plus ou moins grande des filaments génitaux.

Les caractères des mâles sont un peu variables mais les variations se montrent groupées de telle sorte qu'on semble autorisé à parler de types distincts :

1) Le type de Yougoslavie (représenté par les trois mâles étudiés) se présente avec un organe intromittent plus long et plus mince, un tubercule subapical plus petit, des filaments génitaux moins longs.

2) Le type de Bucarest, à organe intromittent plus court, à tubercule subapical plus développé et plus éloigné de l'extrémité, à filaments génitaux excessivement longs. C'est le type identique à celui décrit par Parrot à Bokhara.

Adler et Theodor ont d'ailleurs, avant nous, remarqué combien l'espèce chinensis est variable et ils ont figuré quelques caractères distinctifs revêtus par l'aile ou par l'organe intromittent, pour les exemplaires d'Alep, de Rosh Pinah ou de Chine. Il serait intéressant de constater si ces variations ne sont pas là encore en corrélation mutuelle, et ne convergent pas vers certains types constants. Nous sommes convaincus qu'en récoltant du matériel dans ce but,

(1) Nous donnerons prochainement, dans un mémoire que nous préparons avec Mme Nitzulescu, une description détaillée des phlébotomes de ce gîte. 
on pourra rendre un grand service à celui qui tentera de démembrer cette espèce si répandue.

\section{Bibliographie}

AdLer et Theodor. - The distribution of sandflies and leishmaniasis in Palestine, Syria and Mesopotamia. Annals of tropical Medecine and Parasitology, XXIII, 1929, p. 269-306.

Newstead (R.). - On the genus Phlebotomus. Part. III, Phlebotomus major, var. chinensis. Bull. of entomological research, VII, 1916, p. 191.

Nitzulescu (V.). - Contribution à l'étude des Phlébotomes de Roumanie. Annales de Parasitologie, VII, 1929, p. 430-437.

Patton et Hinde. - Notes on the species of Sandflies (genus Phlebotomus) of North China. Proceedings of the royal Society, B, C, 1926.

PARrot (L.). - Sur quelques phlébotomes de la Bokhara. Archives de l'Institut Pasteur d'Algérie, VI, 1928, p. 26-34.

Perfiliev (P.). - Zur vergleichende Anatomie von Phlebotomus. Zeitschrift für Parasitenkunde, I, 1928.

Sinton (J.). - The synonymy of the asiatic species of Phlebotomus. Indian Journal of medical research, XVI, 1928, p. 297-324.

Sinton (J.) et Barraud (J.). - Improved methods for the identification of some species of Phlebotomus used in experimental work. Indian Journal of medical research, XVI, 1928, p. 325-331.

Laboratoire de parasitologie de la Faculté de médecine de Bucarest. 